\title{
Comparison of Short Versus Long Esophageal Myotomy in Cases With Idiopathic Achalasia: A Randomized Controlled Trial
}

\author{
Zaheer Nabi, ${ }^{*}$ Mohan Ramchandani, Mahiboob Sayyed, Radhika Chavan, Santosh Darisetty, Rajesh Goud, \\ H V V Murthy, and D Nageshwar Reddy \\ Asian Institute of Gastroenterology, Hyderabad, Telangana, India
}

\section{Background/Aims}

Per-oral endoscopic myotomy (POEM) is an established treatment for achalasia. The technique of POEM is still evolving and the impact of length of esophageal myotomy on the outcomes of POEM is not known. In this study, we aim to compare the outcomes of short $(3 \mathrm{~cm})$ versus long $(6 \mathrm{~cm}$ and above) esophageal myotomy in patients undergoing POEM for achalasia cardia.

\section{Methods}

Consecutive patients with idiopathic achalasia (type I and II) were randomized to receive short $(3 \mathrm{~cm})$ or long esophageal myotomy $(\geq 6 \mathrm{~cm})$. Both groups were compared for clinical success, operative time, adverse events, and gastroesophageal reflux disease (GERD).

\section{Results}

Seventy-one consecutive patients with type I and II achalasia underwent POEM with short $(n=34)$ or long $(n=37)$ esophageal myotomy techniques. Mean length of esophageal myotomy in short and long groups was $2.76 \pm 0.41$ and $7.97 \pm 2.40$, respectively $(P<0.001)$. Mean operative time was significantly shorter in short myotomy group $(44.03 \pm 13.78$ minutes and $72.43 \pm 27.28$ minutes, $P<0.001$ ). Clinical success was comparable in both arms at 1 -year (Eckardt score $0.935 \pm 0.929$ vs $0.818 \pm 0.983, P=0.627$ ). Improvement in objective parameters including integrated relaxation pressure and barium column height at 5 minutes was similar in both groups. GERD was detected in $50.88 \%$ patients with no significant difference in short and long myotomy groups (44.44\% vs $56.67 \%, P=0.431)$.

\section{Conclusions}

A short esophageal myotomy is non-inferior to long myotomy with regards to clinical success, adverse events, and GERD in cases with type I and II achalasia. Reduced operating duration favors short esophageal myotomy in these patients.

\section{(J Neurogastroenterol Motil 2021;27:63-70)}

Key Words

Endoscopy; Esophageal achalasia; Gastroesophageal reflux; Myotomy

Received: February 10, 2020 Revised: March 13, 2020 Accepted: March 29, 2020

(a) This is an Open Access article distributed under the terms of the Creative Commons Attribution Non-Commercial License (http://creativecommons. org/licenses/by-nc/4.0) which permits unrestricted non-commercial use, distribution, and reproduction in any medium, provided the original work is properly cited.

*Correspondence: Zaheer Nabi, MD, DNB

Asian Institute of Gastroenterology, 6-3-661, Somajiguda, Hyderabad, Telangana 500082, India Tel: +91-40-2337-8888, Fax: +91-40-2332-4255, E-mail: zaheernabi1978@gmail.com 


\section{Introduction}

Achalasia cardia is a neurodegenerative disease characterized by defective esophageal motility and failure of the lower esophageal sphincter (LES) to relax in response to food bolus. ${ }^{1}$ The currently available treatment modalities including pneumatic dilatation, laparoscopic Heller's myotomy, and per-oral endoscopic myotomy (POEM) aim to palliate the symptoms of achalasia by reducing the resistance at the gastroesophageal junction (GEJ). POEM is a relatively new endoscopic treatment modality for achalasia with excellent results in short and mid-term follow-up studies. ${ }^{2-4}$ However, the technique of POEM has largely remained unchanged since its introduction about a decade ago. ${ }^{5}$ Traditionally, a variable extent of esophageal myotomy ranging from $6 \mathrm{~cm}$ to $10 \mathrm{~cm}$ and $2 \mathrm{~cm}$ to 3 $\mathrm{cm}$ of gastric myotomy is performed during POEM. Meanwhile, the significance of gastric extent of myotomy has been demonstrated in previous studies. ${ }^{6-8}$ The clinical relevance of esophageal myotomy length is not well known. A long length of esophageal myotomy has been recommended in type III achalasia and other non-achalasia spastic esophageal motility disorders such as distal esophageal spasm and Jackhammer esophagus. ${ }^{9,10}$ In contrast to spastic motility disorders, a short esophageal myotomy may be sufficient in cases with type I and II achalasia. ${ }^{11}$ However, there is no randomized trial assessing the efficacy of a short esophageal myotomy in this subgroup of patients.

In this study, we aim to compare the clinical outcomes of short esophageal myotomy versus long esophageal myotomy in patients with idiopathic type I and II achalasia.

\section{Materials and Methods}

\section{Study Design}

This was a prospective, randomized, double blind controlled study conducted at a tertiary care center from June, 2017 to March, 2019. Consecutive patients with confirmed diagnoses of type I and II achalasia who underwent POEM were included in the study. The exclusion criteria specific to this study were age $<18$ years, type III achalasia, non-achalasia spastic motility disorders, sigmoid type achalasia, and a history of previous Heller's myotomy. Standard exclusion criteria for POEM procedure comprised of contraindication to general anesthesia, coagulopathy (international normalized ratio $>1.5$, platelet count $<50000 / \mu \mathrm{L}$ ), portal hypertension, pregnancy, breast feeding women, and diffuse scarring in the esophagus due to previous surgery or radiation.

The study was approved by institutional review board committee (AIG/IEC29/05.2017-15) and registered with Clinical Trials Registry (NCT 03186248).

\section{Calculation of Sample Size}

The primary outcome of the study was to compare the clinical success in both groups. It was assumed the clinical success of conventional POEM is $94 \%$ at 1 -year based on a previously published study. ${ }^{3}$ We postulated that a short esophageal myotomy would be non-inferior to long esophageal myotomy specifying a priori a non-inferiority margin of $15 \%$. The sample size calculated was 64 (32 per group) to demonstrate non-inferiority with type 1 error as 0.05 and $80 \%$ power. Considering a $10 \%$ drop out rate during the follow-up, the number of cases to be enrolled was calculated to be 70 (35 in each group).

\section{Randomization Method}

Consecutive eligible patients were randomized in a 1:1 ratio to short or long myotomy groups. Randomization was done according to the computer-generated algorithm. A clinical research coordinator prepared the sealed randomization envelops and handed over to the operating endoscopists before the beginning of the procedure. Written informed consent was obtained from all participants. In this study, the participants and the care-provider assessing the clinical response to the POEM procedure at 1-year were blinded to the allocation.

\section{Per-oral Endoscopic Myotomy Technique}

POEM was performed by 3 experienced endoscopists (Z.N., M.R., and D.N.R.) each with an experience of $>100$ POEM procedures. All procedures were performed under general anesthesia with the patients in the supine position.

POEM was performed by anterior (1-2 O'clock) route in all cases. The steps of POEM procedure were as follows: (1) submucosal injection using a solution of saline mixed with indigocarmine dye (Fig. 1A), (2) vertical mucosal incision measuring $2 \mathrm{~cm}$ to 2.5 $\mathrm{cm}$ in length, (3) submucosal tunneling extending up to $2 \mathrm{~cm}$ to 4 $\mathrm{cm}$ below the GEJ (Fig. 1B and 1C), (4) myotomy extending from $1 \mathrm{~cm}$ to $2 \mathrm{~cm}$ below the distal edge of mucosal incision until the lower end of the tunnel (Fig. 1D and 1E), and (5) closure of the mucosal incision with several endoclips (Fig. 1F). Triangular tip knife (KD-645L; Olympus, Tokyo, Japan) with integrated water jet function was utilized for the entire procedure including mucosal incision, submucosal tunneling, and myotomy (Spray Coag $50 \mathrm{~W}$, 
effect 2). Hemostasis was achieved with coagulation forceps (Coagrasper G, FD-412LR; Olympus) in soft coagulation mode (80 W, effect 4).

The important technical differences in the short and long myotomy groups were as follows. The length of esophageal myotomies ( $\leq 3 \mathrm{~cm}$ vs $\geq 6 \mathrm{~cm}$ ) were different in both groups. Whereas, the length of gastric myotomies were similar $(2-4 \mathrm{~cm})$ in either group. In the short myotomy group, the mucosal incision was performed at about 5-6 cm above the GEJ. The length of esophageal myotomy was $2 \mathrm{~cm}$ to $3 \mathrm{~cm}$ and the entire extent of the myotomy was full thickness in the short myotomy group. Whereas in the long myotomy group, the mucosal incision was performed at least $8 \mathrm{~cm}$ above the GEJ and the length of esophageal myotomy was $\geq 6 \mathrm{~cm}$. Selective circular myotomy was performed in the upper part of the tunnel and full thickness myotomy was performed from $2-3 \mathrm{~cm}$ above the GEJ until the lower end of the tunnel. The length of gastric myotomy was kept similar in both groups ie, $2-4 \mathrm{~cm}$. The extent of esophageal and gastric myotomies were assessed using scope withdrawal, and fluoroscopy (Fig. 2A and 2B). In cases with uncertainty regarding the gastric length of myotomy, double scope method was used for
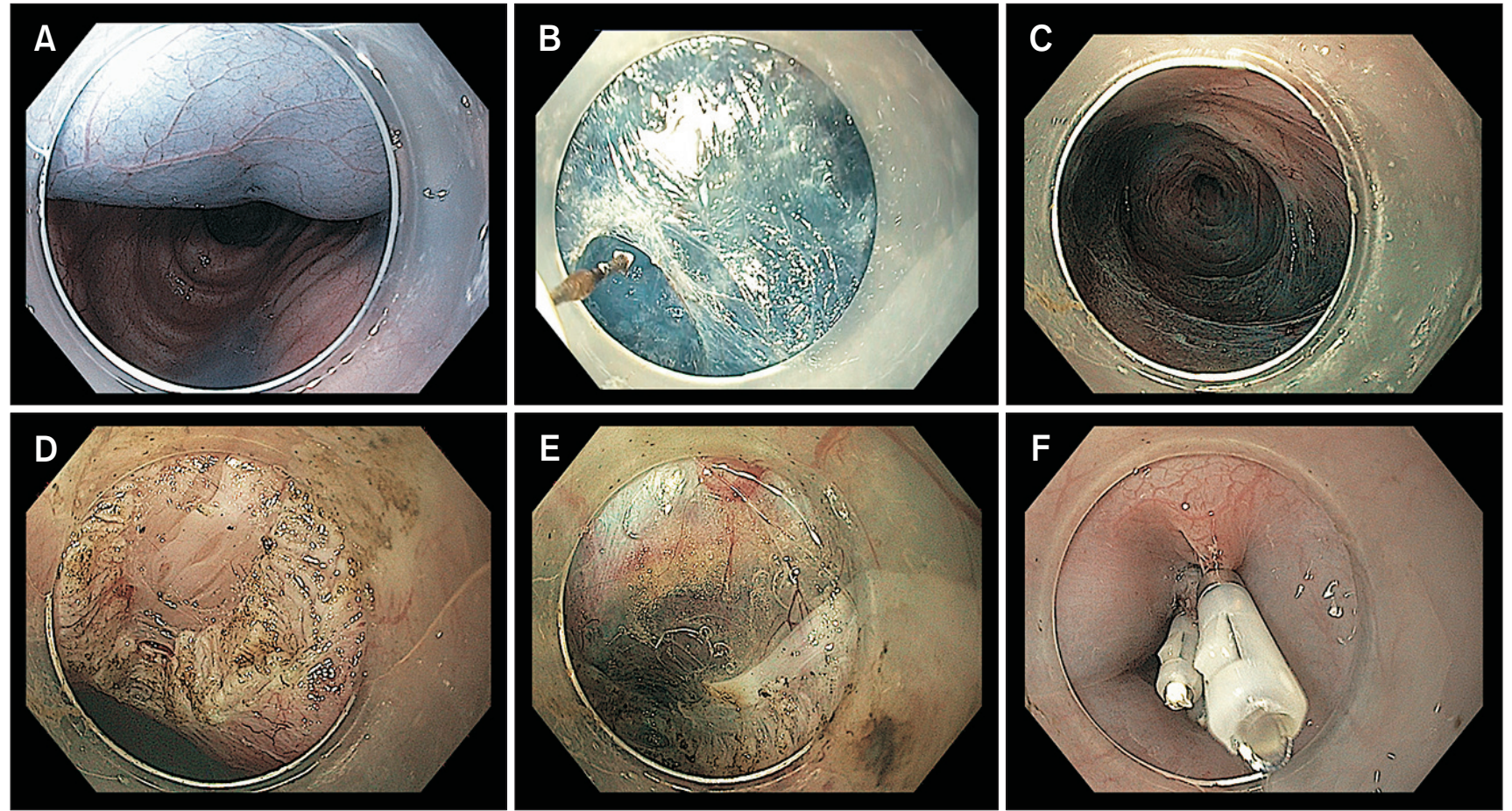

Figure 1. Technique of anterior per-oral endoscopic myotomy. (A) Submucosal injection to raise a mucosal bleb. (B) Submucosal tunneling using a triangular tip knife. (C) Completion of submucosal tunneling. (D) Selective circular myotomy in upper part of submucosal tunnel. (E) Full thickness myotomy in the lower end of tunnel. (F) Closure of mucosal incision with multiple endoclips.
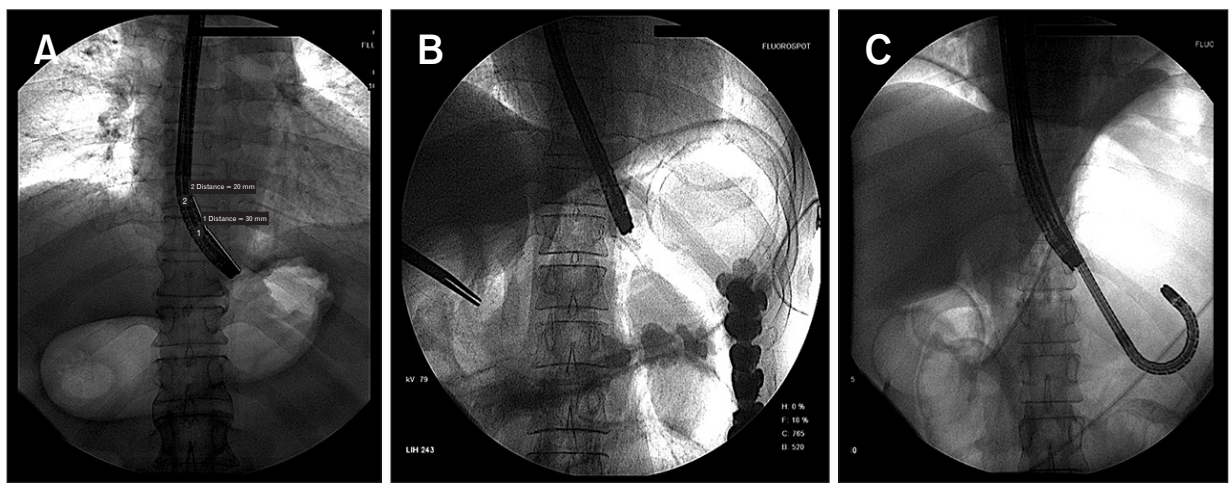

Figure 2. Use of fluoroscopy to determine the length of esophageal and gastric myotomies. (A) Fluoroscopic view depicting the location of endoscope tip at the gastroesophageal junction. (B) Fluoroscopic determination of gastric extent of myotomy. (C) Double scope technique to confirm the gastric length of myotomy. 
confirmation (Fig. 2C).

After POEM procedure, all the patients were kept nil per oral for 24-hours. Timed barium esophagogram was performed on the next day of POEM and oral liquids allowed. Subsequently, soft pureed diet was allowed on day 3 of POEM procedure before discharge.

\section{Follow-up Protocol}

Patients were followed-up at 1, 3, 6, and 12 months after the POEM procedure. Assessment of clinical efficacy using the Eckardt score was performed at each of the follow-up visit. Objective evaluation of success was analyzed with high-resolution esophageal manometry and timed barium esophagogram at 1 month. Gastroesophageal reflux disease (GERD) was assessed using esophagogastroduodenoscopy and 24-hour $\mathrm{pH}$ impedance study at 3 months.

\section{Primary and Secondary Outcomes}

The primary aim of the study was comparison of clinical success (Eckardt $\leq 3)$ of POEM procedure using short versus long esophageal myotomy techniques at 1-year follow-up. The secondary outcome measures included difference in operating times, intra-operative adverse events, GERD, barium column height at 5 minutes, and reduction in integrated relaxation pressures at 3 months.

\section{Definitions}

Short esophageal myotomy: an esophageal myotomy length that did not exceed $3 \mathrm{~cm}$ was defined as short myotomy.

Long esophageal myotomy: an esophageal length of at least 6 $\mathrm{cm}$ was defined as long myotomy.

Operating duration: the time taken from mucosal incision to the completion of closure of incision was regarded as procedure duration.

Adverse events: events requiring an additional procedure such as needle drainage for capno-peritoneum, temporary cessation of procedure due to accumulation of retroperitoneal $\mathrm{CO}_{2}$, mucosal injuries requiring closure with endoclips, and intra-procedural or post-procedural events leading to prolongation of hospital stay were considered as adverse events. ${ }^{12}$

Clinical success: Eckardt score $\leq 3$ was used to define clinical success. Eckardt score is a composite score based on 4 variables including dysphagia, regurgitation, chest pain, and weight loss.

Table 1. Baseline Characteristics of Patients in Short and Long Myotomy Groups

\begin{tabular}{lccc}
\hline Baseline characteristics & Long myotomy & Short myotomy & $P$-value \\
\hline Number of patients & 37 & 34 & \\
Age (yr) & $41.3 \pm 14.4$ & $40.1 \pm 16.8$ & 0.747 \\
Sex & & & 0.342 \\
$\quad$ Males & $24(64.86)$ & $18(52.94)$ & \\
$\quad$ Females & $13(35.14)$ & $16(47.06)$ & \\
Duration of illness (yr) & $3(1.0-5.0)$ & $3(1.5-4.7)$ & 1.000 \\
Eckardt score & $6.75 \pm 1.32$ & $6.02 \pm 1.33$ & 0.023 \\
Type of achalasia & & & $>0.99$ \\
$\quad$ Type I & $13(35.14)$ & $12(35.29)$ & \\
$\quad$ Type II & $24(64.86)$ & $22(64.71)$ & \\
Prior pneumatic & $9($ single: 6, & $12($ single: 7, & 0.436 \\
$\quad$ dilatation & two: 3$)$ & two: 5$)$ & \\
Integrated relaxation & $28.50 \pm 11.01$ & $26.40 \pm 13.93$ & 0.482 \\
pressure (mmHg) & & & \\
Barium column at & $11.21 \pm 5.36$ & $12.99 \pm 5.40$ & 0.168 \\
5 min (cm) & & & \\
\hline
\end{tabular}

Data are presented as mean $\pm \mathrm{SD}, \mathrm{n}(\%)$, or median (interquartile range).

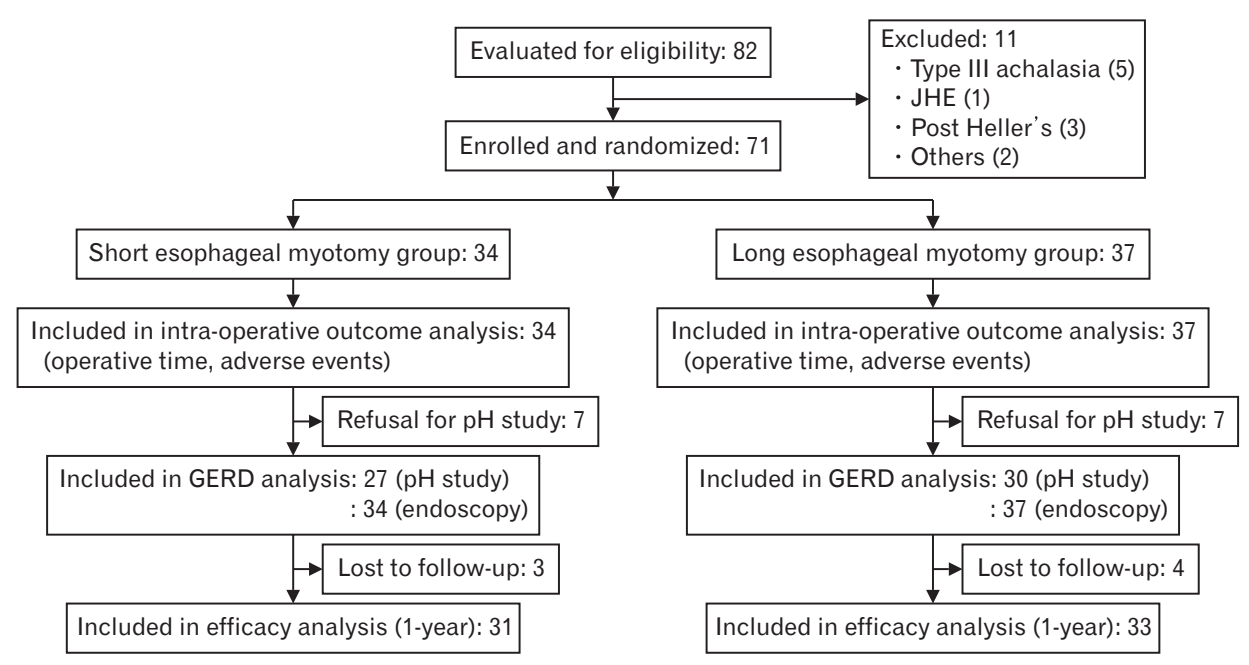

Figure 3. Flow diagram. JHE, Jackhammer esophagus; GERD, gastroesophageal reflux disease. 
GERD: Lyon consensus criteria were used to define GERD according to 24-hour $\mathrm{pH}$ study. ${ }^{13}$ An acid exposure time of $>6 \%$ and a DeMeester score $>14.72$ were considered consistent with the diagnosis of GERD. Endoscopic esophagitis was graded according to the Los Angeles classification for reflux esophagitis.

\section{Statistical Methods}

The data for the present study were collected on a pre-designed format. The primary outcome of the study included the noninferiority of short esophageal myotomy at 1-year follow-up according to the per-protocol method. The population in the per-protocol approach consisted of patients who completed 1-year follow-up for the assessment of clinical efficacy. Short esophageal myotomy was considered noninferior to the long myotomy if the two-sided $95 \%$ confidence interval for the mean difference between both the approaches was less than $15 \%$ as defined previously.

The data was expressed as mean \pm standard deviation (SD) or median and interquartile range (IQR) for continuous variables. The categorical variables were expressed as \% frequency distribution. The Students $t$ test, median test and Fisher's exact test were applied for comparison between the 2 groups. A two-sided $P$-value $<0.05$ was considered as statistically significant. The analysis was carried out by using Statistical package for social sciences (SPSS version 20.0; IBM Corp, Armonk, NY, USA).

\section{Results}

A total of 71 patients with type I and II achalasia were randomized into short ( $\mathrm{n}=34)$ and long $(\mathrm{n}=37)$ esophageal myotomy groups (Fig. 3). Of these, 64 patients completed 1-year follow-up and were included in the analysis of clinical success. The baseline demographic characteristics including age, sex, type of achalasia, and history of previous pneumatic dilatations were similar in both groups. Objective parameters at baseline including pre-POEM Eckardt score, barium column height at 5 minutes, and integrated relaxation pressures were not significantly different in both groups (Table 1).

\section{Peri-operative Outcomes}

POEM was successfully performed in all eligible patients in both groups (Technical success $=100 \%$ ). The mean length of esophageal myotomy was significantly smaller in the short myotomy group (mean $\pm \mathrm{SD}: 2.76 \pm 0.41 \mathrm{~cm}$ vs $7.97 \pm 2.40 \mathrm{~cm}, P<0.001)$. There was no significant difference in the length of gastric myotomies in both groups (mean $\pm \mathrm{SD}: 2.70 \pm 0.73 \mathrm{~cm}$ vs $2.84 \pm 0.63 \mathrm{~cm}, P=0.389$ ).
Intraprocedural fluoroscopy was utilized in all cases to confirm esophageal and gastric extent of myotomy. Whereas, the double scope method was utilized in 3 cases to confirm the gastric extent of myotomy.

The mean operating duration was significantly less in the short esophageal myotomy group $(44.03 \pm 13.78$ minutes vs $72.43 \pm$ 27.28 minutes, $P<0.001)$. The median duration of hospitalization was similar in both groups (median [IQR]: 3 [2-4] days vs 3 [2-4] days) (Table 2).

\section{Adverse Events}

There were no major adverse events. Minor intra-operative adverse events were encountered in $8(11.27 \%)$ patients including capnoperitoneum requiring needle drainage in 6 patients and mucosal injuries requiring closure with endoclips in 2 patients. The incidence of insufflation related adverse events and mucosal injuries were not significantly different between the 2 groups (short $11.76 \%$ vs long $10.81 \%, P=1.000)$. Other insufflation related events including subcutaneous emphysema and retroperitoneal $\mathrm{CO}_{2}$ were similar in both groups (Table 2).

\section{Clinical Success}

Clinical success was recorded in all participants $(\mathrm{n}=71$, $100.00 \%$ at 1 -month follow-up. There was significant and similar

Table 2. Peri-operative Outcomes in Both Comparison Groups

\begin{tabular}{|c|c|c|c|}
\hline $\begin{array}{c}\text { Procedure } \\
\text { characteristics }\end{array}$ & $\begin{array}{l}\text { Long myotomy } \\
\qquad(\mathrm{n}=37)\end{array}$ & $\begin{array}{l}\text { Short myotomy } \\
\qquad(\mathrm{n}=34)\end{array}$ & $P$-value \\
\hline $\begin{array}{l}\text { Length of esophageal } \\
\text { myotomy }(\mathrm{cm})\end{array}$ & $7.97 \pm 2.40$ & $2.76 \pm 0.41$ & $<0.001$ \\
\hline $\begin{array}{l}\text { Length of gastric } \\
\text { myotomy }(\mathrm{cm})\end{array}$ & $2.84 \pm 0.63$ & $2.70 \pm 0.73$ & 0.389 \\
\hline Total operating time & $72.43 \pm 27.28$ & $44.03 \pm 13.78$ & $<0.001$ \\
\hline Clips required & $6.80 \pm 2.03$ & $6.20 \pm 2$ & 0.246 \\
\hline \multicolumn{4}{|l|}{ Insufflation related events } \\
\hline $\begin{array}{l}\text { Subcutaneous } \\
\text { emphysema }\end{array}$ & $4(10.81)$ & $4(11.76)$ & 1.000 \\
\hline $\begin{array}{l}\text { Capnoperitoneum } \\
\text { requiring decompression }\end{array}$ & $3(8.10)$ & $3(8.82)$ & 1.000 \\
\hline Retroperitoneal $\mathrm{CO}_{2}$ & $2(5.40)$ & $4(11.76)$ & 0.417 \\
\hline $\begin{array}{l}\text { Minor bleeding } \\
\text { episodes }\end{array}$ & $17(45.94)$ & $12(35.29)$ & 0.469 \\
\hline $\begin{array}{l}\text { Mucosal injuries } \\
\text { requiring clipping }\end{array}$ & $1(2.70)$ & $1(2.94)$ & 1.000 \\
\hline Hospital stay (day) & $2.81 \pm 0.70$ & $2.82 \pm 0.67$ & 0.951 \\
\hline
\end{tabular}

Data are presented as mean $\pm \mathrm{SD}$ or $\mathrm{n}(\%)$. 
Table 3. Comparison of Clinical Success Between Short and Long Myotomy Groups

\begin{tabular}{lccc}
\hline Success parameters & $\begin{array}{c}\text { Long myotomy } \\
(\mathrm{n}=37)\end{array}$ & $\begin{array}{c}\text { Short myotomy } \\
(\mathrm{n}=34)\end{array}$ & $P$-value \\
\hline Eckardt score & & & \\
$1 \mathrm{mo}$ & $\begin{array}{c}0.405 \pm 0.864 \\
(\mathrm{n}=37)\end{array}$ & $\begin{array}{c}0.412 \pm 0.743 \\
(\mathrm{n}=34)\end{array}$ & 0.971 \\
& $\begin{array}{c}0.818 \pm 0.983 \\
(\mathrm{n}=33)\end{array}$ & $\begin{array}{c}0.935 \pm 0.929 \\
(\mathrm{n}=31)\end{array}$ & 0.627 \\
$1 \mathrm{yr}$ & 96.97 & 93.55 & 0.607 \\
Clinical success & $(84-100)$ & $(85-100)$ & \\
at 1 yr & $7.44 \pm 4.30$ & $8.60 \pm 1.30$ & 0.155 \\
Integrated relaxation & & & \\
pressure (mmHg) & & $1.90 \pm 2.39$ & 0.406 \\
Barium column at & $2.31 \pm 1.71$ & & \\
5 min (cm) & & & \\
\hline
\end{tabular}

Data are presented as mean $\pm \mathrm{SD}$ or $\%(\mathrm{CI})$.

reduction in Eckardt score at 1 month in both groups. At 1-year follow-up, 64 (90.14\%) patients were available for evaluation of clinical success including 31 in short and 33 in long myotomy group. The mean Eckardt scores were similar in short and long myotomy groups $(0.935 \pm 0.929$ vs $0.818 \pm 0.983 ; P=0.627)$ at 1 year. Clinical success was noted in $93.55 \%$ (84-100) in the short myotomy and $96.97 \%$ (85-100) in the long myotomy group. Objective improvement in integrated relaxation pressure and reduction in height of barium column at 5 minutes was equivalent in both groups (Table 3).

\section{Gastroesophageal Reflux Disease}

GERD was analyzed in all 71 participants with gastroscopy. Endoscopic evidence of erosive esophagitis was found in 11 (32.35\%) and 18 (48.65\%) patients in short and long myotomy groups, respectively. A total of 57 (80.28\%) patients (short myotomy 27, long myotomy 30) underwent analysis of GERD using 24-hour $\mathrm{pH}$ impedance study. Increased esophageal acid exposure $(>6 \%)$ and high DeMeester scores ( $>14.72)$ were detected in 7 (25.92\%) and 12 (44.44\%) patients in the short myotomy group, respectively. Whereas, 12 (40.00\%) and 17 (56.67\%) patients in the long myotomy group had increased esophageal acid exposure and high DeMeester scores, respectively. There was no significant difference in the incidence of GERD between the 2 groups when evaluated with endoscopy and 24-hour pH impedance study (Table 4).

\section{Discussion}

In this randomized trial, we found that short esophageal my-
Table 4. Gastroesophageal Reflux Disease in Both Groups

\begin{tabular}{cccr}
\hline Reflux parameters & $\begin{array}{c}\text { Long } \\
\text { myotomy }\end{array}$ & $\begin{array}{c}\text { Short } \\
\text { myotomy }\end{array}$ & P-value \\
\hline Esophagogastroduodenoscopy & $\mathrm{n}=37$ & $\mathrm{n}=34$ & \\
Grade A esophagitis & $8(21.62)$ & $5(14.71)$ & 0.546 \\
Grade B esophagitis & $9(24.32)$ & $6(17.65)$ & 0.569 \\
Grade C esophagitis & $1(2.70)$ & $0(0.00)$ & $>0.999$ \\
Grade D esophagitis & $0(0.00)$ & $0(0.00)$ & \\
24-hr pH impedance study & $\mathrm{n}=30$ & $\mathrm{n}=27$ & \\
Total reflux episodes & $71.30 \pm 50.37$ & $61.03 \pm 45.60$ & 0.425 \\
Increased esophageal & $12(40.00)$ & $7(25.92)$ & 0.399 \\
acid exposure $>$ ( $7 \%)$ & & & \\
DeMeester score & $28.49 \pm 33.40$ & $27.00 \pm 51.33$ & 0.896 \\
High DeMeester & $17(56.67)$ & $12(44.44)$ & 0.431 \\
score $(\geq 14.72)$ & & & \\
\hline
\end{tabular}

Data are presented as $\mathrm{n}(\%)$ or mean $\pm \mathrm{SD}$.

otomy was non-inferior to long myotomy in cases with type I and II achalasia. Short myotomy is associated with less operation time.

Third space endoscopy has revolutionized the endoscopic management of various gastrointestinal disorders. ${ }^{14} \mathrm{POEM}$ is the frontier among third space endoscopy procedures and has emerged as a safe and efficacious endoscopic modality for the management of achalasia and other non-achalasia spastic esophageal motility disorders. Since its introduction about a decade ago by Inoue and colleagues, ${ }^{5}$ the technique of POEM is more or less unchanged. Major technical variations in POEM procedures across the globe include orientation of myotomy (anterior vs posterior), thickness of myotomy (full thickness vs partial thickness) and the length of esophageal myotomy (short vs long). The length of gastric myotomy has been shown to affect the efficacy and GERD ${ }^{6-8}$ On the other hand, the impact of esophageal myotomy length on the outcomes of POEM is not well known. In this randomized study, we analyzed the influence of esophageal myotomy length on the outcomes of POEM. Type III achalasia and other spastic esophageal motility disorders were excluded from the study as these disorders require a longer myotomy.

The mean length of esophageal myotomy and the operating times were significantly shorter in the short myotomy group. It is important to note that only the difference in esophageal myotomy length was compared in this study. Gastric length of myotomy should be at least $2 \mathrm{~cm}$ to avoid recurrences. ${ }^{6}$ Therefore, gastric myotomy was performed in similar fashion in both groups. Similar to the results of our study, a short myotomy $(\leq 7 \mathrm{~cm})$ was found to be associated with significantly shorter procedure duration (49.2 \pm 19.2 minutes vs $64.9 \pm 24.4$ minutes,$P=0.010$ in a case- 
control study published in abstract form. ${ }^{15}$ Shorter operating time can potentially reduce the overall cost of the procedure related to the occupancy of the endoscopy suite. In addition, insufflation related events have been shown to be fewer in cases with shorter procedure duration. ${ }^{16}$ However, there was no significant difference in the gas related adverse events in the current study presumably due to insufficient sample size with respect to difference in adverse events.

There was no significant difference between the 2 groups with regards to clinical success at 3 months and 1 year, respectively. Objective parameters of clinical success including post-POEM integrated relaxation pressures and emptying on timed barium swallow study were similar in the 2 groups. Short myotomy has been shown to be effective in 2 non-randomized studies with short follow-up duration. ${ }^{11,15}$ This implies that the length of esophageal myotomy has minimal impact on the outcomes of POEM in cases with type I and II achalasia. However, longer follow-up studies are required to confirm our observation.

GERD was detected in $40.84 \%$ and $50.88 \%$ cases utilizing endoscopy and 24-hour $\mathrm{pH}$ impedance study, respectively. High incidence of GERD in our study stands in concordance with published studies. ${ }^{17-19}$ There was no difference in the incidence of GERD between both groups. This finding supports the notion that the length of esophageal myotomy has little, if any, bearing on the incidence of GERD. On the contrary, the length of gastric myotomy has been shown to affect the incidence of severe esophagitis in a recent study. ${ }^{7,8}$ In the present study, the length of gastric myotomies was similar in both groups explaining the similarity in the incidence of GERD.

The implications of our study are manifold. First, a long esophageal myotomy $(>6 \mathrm{~cm})$ has no clinically significant impact on the outcomes of POEM in type I and II achalasia. Moreover, reduced operating time favors a short esophageal myotomy in these cases. Shorter operating times may reduce the incidence of insufflation related adverse events, as well as the overall expense incurred due to occupancy of the endoscopy suite. However, we did not find a difference in adverse events, presumably because our study was not powered to see such a difference. In addition, we did not analyze the cost benefits of a short esophageal myotomy.

The main strength of this study is that it is a prospective, randomized study. Clinical success was evaluated and compared objectively in both groups. Comprehensive evaluation of GERD was performed using endoscopy and 24-hour $\mathrm{pH}$ impedance study. However, certain drawbacks are noteworthy. In the majority of cases, the length of esophageal and gastric myotomy was assessed using fluoroscopy and the scope withdrawal method. Double scope technique was not utilized to quantify gastric myotomy as recommended by some experts. ${ }^{7,20}$ Therefore, we may have marginally underestimated or overestimated the length of esophageal and gastric myotomy. The present study was a non-inferiority trial and it may be premature to conclude the superiority of any one approach over the other. A larger sample size with longer follow-up duration may be required to demonstrate the same conclusions. We did not analyze the difference in outcomes with respect to the sub-type of achalasia (type I and II) as the present study was not powered for the same outcomes. Therefore, the relative difference in outcomes between type I and II achalasia needs to be evaluated in prospective studies.

In conclusion, a short esophageal myotomy provides similar clinical success with the added advantage of reduced procedure duration as compared to long esophageal myotomy.

\section{Financial support: None.}

\section{Conflicts of interest: None.}

Author contributions: Zaheer Nabi and H V V Murthy: analysis and interpretation of data; Mahiboob Sayyed, Radhika Chavan, and Rajesh Goud: collection of data; and Mohan Ramchandani, Santosh Darisetty, and D Nageshwar Reddy: critical inputs to the manuscript.

\section{References}

1. Nabi Z, Ramchandani M, Reddy DN, et al. Per oral endoscopic myotomy in children with achalasia cardia. J Neurogastroenterol Motil 2016;22:613-619.

2. Inoue $H$, Sato $H$, Ikeda $H$, et al. Per-oral endoscopic myotomy: a series of 500 patients. J Am Coll Surg 2015;221:256-264.

3. Nabi Z, Ramchandani M, Chavan R, et al. Per-oral endoscopic myotomy for achalasia cardia: outcomes in over 400 consecutive patients. Endosc Int Open 2017;5:E331-E339.

4. Youn YH, Minami H, Chiu PW, Park H. Peroral endoscopic myotomy for treating achalasia and esophageal motility disorders. J Neurogastroenterol Motil 2016;22:14-24.

5. Inoue H, Minami H, Kobayashi Y, et al. Peroral endoscopic myotomy (POEM) for esophageal achalasia. Endoscopy 2010;42:265-271.

6. Oelschlager BK, Chang L, Pellegrini CA. Improved outcome after extended gastric myotomy for achalasia. Arch Surg 2003;138:490-495; discussion 495-497.

7. Inoue H, Shiwaku H, Kobayashi Y, et al. Statement for gastroesophageal reflux disease after peroral endoscopic myotomy from an international multicenter experience. Esophagus 2020;17:3-10.

8. Grimes KL, Bechara R, Shimamura Y, Ikeda H, Haruhiro I. Gastric myotomy length affects severity but not rate of post-procedure reflux: 
3-year follow-up of a prospective randomized controlled trial of doublescope per-oral endoscopic myotomy (POEM) for esophageal achalasia. Surg Endosc 2020;34:2963-2968.

9. Khashab MA, Messallam AA, Onimaru M, et al. International multicenter experience with peroral endoscopic myotomy for the treatment of spastic esophageal disorders refractory to medical therapy (with video). Gastrointest Endosc 2015;81:1170-1177.

10. Khashab MA, Familiari P, Draganov PV, et al. Peroral endoscopic myotomy is effective and safe in non-achalasia esophageal motility disorders: an international multicenter study. Endosc Int Open 2018;6:E1031E1036.

11. Wang J, Tan N, Xiao Y, et al. Safety and efficacy of the modified peroral endoscopic myotomy with shorter myotomy for achalasia patients: a prospective study. Dis Esophagus 2015;28:720-727.

12. Nabi Z, Reddy DN, Ramchandani M. Adverse events during and after per-oral endoscopic myotomy: prevention, diagnosis, and management. Gastrointest Endosc 2018;87:4-17.

13. Gyawali CP, Kahrilas PJ, Savarino E, et al. Modern diagnosis of GERD: the Lyon Consensus. Gut 2018;67:1351-1362.

14. Nabi Z, Reddy DN, Ramchandani M. Recent advances in third-space endoscopy. Gastroenterol Hepatol (NY) 2018;14:224-232.
15. Tang X, Deng Z, Gong W, Jiang B. Peroral endoscopic short versus long myotomy for the treatment of achalasia: a case-control study. Gastrointest Endosc 2015;5:AB117-AB118.

16. Khashab MA, El Zein M, Kumbhari V, et al. Comprehensive analysis of efficacy and safety of peroral endoscopic myotomy performed by a gastroenterologist in the endoscopy unit: a single-center experience. Gastrointest Endosc 2016;83:117-125.

17. Kumbhari V, Familiari P, Bjerregaard NC, et al. Gastroesophageal reflux after peroral endoscopic myotomy: a multicenter case-control study. Endoscopy 2017;49:634-642.

18. Repici A, Fuccio L, Maselli R, et al. GERD after per-oral endoscopic myotomy as compared with heller's myotomy with fundoplication: a systematic review with meta-analysis. Gastrointest Endosc 2018;87:934943, e18.

19. Nabi Z, Ramchandani M, Reddy DN. Per-oral endoscopic myotomy and gastroesophageal reflux: where do we stand after a decade of "POETRY"? Indian J Gastroenterol 2019;38:287-294.

20. Khashab MA, Kumbhari V, Azola A, et al. Intraoperative determination of the adequacy of myotomy length during peroral endoscopic myotomy (POEM): the double-endoscope transillumination for extent confirmation technique (DETECT). Endoscopy 2015;47:925-928. 